\title{
HOSPITALIZACIONES POR FRACTURA FEMORAL ENTRE ANCIANOS EN RORAIMA, BRASIL
}

\section{COMUNICACIÓN}

BORGHETTI, Gina ${ }^{1}$

BORGHETTI, Gina. Hospitalizaciones por fractura femoral entre ancianos en Roraima, Brasil. Revista Científica Multidisciplinar Núcleo do Conhecimento. Año. 07, Ed. 01, Vol. 02, págs. 111-119. Enero 2022. ISSN: 2448-0959, Enlace de acceso: https://www.nucleodoconhecimento.com.br/salud/fractura-femoral, DOI: 10.32749/nucleodoconhecimento.com.br/salud/fractura-femoral

\section{RESUMEN}

Introducción: Las fracturas femorales en ancianos aumentan el riesgo de morbilidad y mortalidad, y suelen ser necesarias para la hospitalización. Debido a la relevancia del tema en salud pública y la falta de información sobre la situación en el estado en el período comprendido entre 2015 y 2019, surgió la siguiente pregunta: Según el grupo de edad y el género, ¿cuál es el perfil de los ingresos hospitalarios por fractura femoral entre los ancianos en Roraima en este período? Objetivo: Describir los ingresos hospitalarios por fractura femoral entre ancianos, entre 2015 y 2019 en el Estado de Roraima, Brasil. Método: Se trata de un estudio descriptivo, con enfoque cuantitativo, utilizando datos del Sistema de Información Hospitalaria[2] del Sistema Único de Salud[3] en el período de 2015 a 2019. Para los análisis de frecuencia se utilizaron códigos relacionados con la fractura femoral según la 10 a revisión de la Clasificación Internacional de Enfermedades, grupo de edad, sexo y muertes hospitalarias. Resultados: Se observó que el sexo femenino fue el más prevalente en las hospitalizaciones por fractura de fémur (56,4\%). Estratificado por grupo de edad, 80 años y más representó el 38,7\% de las hospitalizaciones, en su mayoría mujeres. Este mismo perfil se observó cuando se analizaron las

\footnotetext{
${ }^{1}$ Doctor en Biología Celular y Molecular, Máster en Biología Celular y Molecular, Grado en Fisioterapia. ORCID: https://orcid.org/0000-0002-2922-9090.

RC: 105706

Disponible: https://www.nucleodoconhecimento.com.br/salud/fractura-femoral
} 
hospitalizaciones por fractura femoral con localización proximal. Entre las hospitalizaciones con resultado de muerte por fractura femoral, hubo predominio entre las mujeres de 80 años y más (66,7\%). Consideraciones finales: En este estudio, se observó que las hospitalizaciones y muertes hospitalarias por fractura femoral fueron más prevalentes en mujeres octogenarias. Las estrategias de salud, como la prevención de caídas, deben dirigirse a la población de edad avanzada, especialmente a las mujeres, que son las más afectadas por esta enfermedad.

Palabras clave: Fracturas femorales, Hospitalización, Ancianos, Salud Pública.

\section{INTRODUCCIÓN}

La transición demográfica ocurre en Brasil simultáneamente con el envejecimiento creciente y progresivo de la población, afectando los servicios de salud y las condiciones socioeconómicas de la sociedad (TRAVASSO et al., 2020). Los factores relacionados con el envejecimiento en asociación con la fragilidad, la osteoporosis y la presencia de comorbilidades aumentan la aparición de fracturas entre los ancianos (PETERLE et al., 2019; WU et al., 2019). Un estudio realizado en Brasil en 2013 mostró una prevalencia de tres enfermedades simultáneas en la población de 60 años o más. Otros factores como la pérdida de la función muscular y el bajo rendimiento físico también son indicativos de eventos desfavorables en esta población (PETERLE et al., 2019).

La mayoría de las fracturas femorales en los ancianos son secundarias a caídas accidentales, y el tratamiento quirúrgico es el procedimiento más utilizado (BORTOLON et al., 2011; ARMOND et al., 2014). En Brasil, la prevalencia de al menos una caída en 2019 varió de $25,1 \%$ a 37,07\% (SANTOS et al., 2021). Sin embargo, este evento no debe verse como parte del envejecimiento natural (PORTO et al., 2019). Las caídas podrían prevenirse con intervenciones a través de ejercicios físicos guiados, identificación temprana de factores de riesgo y el tratamiento de la osteoporosis, especialmente en mujeres posmenopáusicas (BEER y GILES, 2005). 
La investigación indica que el aumento de las fracturas femorales está relacionado con el avance de la edad y el sexo (BORTOLON et al., 2011). Estas fracturas pueden ocurrir en diferentes áreas anatómicas del fémur, como la región proximal, la diáfisis y la región distal (EDELMUTH et al., 2018). Los estudios indican que las fracturas localizadas en el fémur proximal, principalmente debido a la osteoporosis, presentan un mayor riesgo de morbilidad y mortalidad y fracturas recurrentes (CHEN et al., 2018; CARMO y GOMES, 2008).

Las fracturas femorales aumentan el riesgo de mortalidad entre los ancianos, especialmente si se asocian con factores como el grupo de edad, las comorbilidades, el sexo y la presencia de deterioro cognitivo (PETERLE et al., 2019; NETO et al., 2011). Se demostró que un año después del tratamiento quirúrgico, alrededor del $50 \%$ de los ancianos presentaron dificultades en las actividades diarias y la deambulación (MACEDO et al., 2019). Por lo general, tienen una autonomía reducida y pérdida de calidad de vida (MACEDO et al., 2019; NASCIMENTO, 2019). Adicionalmente, pueden causar impactos psicosociales y generar costos financieros adicionales para el sistema de salud y familiar, ya que estos ancianos generalmente requieren hospitalización y rehabilitación más prolongadas (SANTOS et al., 2021; OLIVEIRA y BORBA, 2017).

Las fracturas femorales en los ancianos aumentan el riesgo de morbilidad y mortalidad y generalmente son necesarias para la hospitalización. La información sobre los ingresos hospitalarios puede ayudar en el conocimiento de esta situación sanitaria y en las estrategias de prevención para esta población. Debido a la relevancia del tema en salud pública y la falta de información sobre la situación en el estado en el período comprendido entre 2015 y 2019, surgió la siguiente pregunta: Según el grupo de edad y el género, ¿cuál es el perfil de los ingresos hospitalarios por fractura femoral entre los ancianos en Roraima en este período?

En vista del contexto presentado, este estudio tiene como objetivo describir los ingresos hospitalarios por fractura femoral entre los ancianos, entre 2015 y 2019 en el Estado de Roraima, Brasil. 


\section{MÉTODO}

Se trata de un estudio descriptivo con enfoque cuantitativo. El banco de datos fue recolectado del Sistema de Información Hospitalaria del Sistema Único de Salud (SIH/SUS), que tiene su origen en las Autorizaciones de Ingreso Hospitalario (AlH'S) del Departamento de Informática del SUS (DATASUS). La base de datos fue seleccionada por Unidad de la Federación (Roraima) y año/mes de procesamiento (período comprendido entre enero de 2015 y diciembre de 2019). Para describir los ingresos hospitalarios por fracturas de fémur se seleccionaron las siguientes variables: Capítulos de la Clasificación Internacional de Enfermedades (CIE-10), Defunciones (con muerte, sin muerte), Grupo de edad (60 a 69 años, 70 a 79 años y 80 años y más) y Género (femenino, masculino). Se analizaron las AlH'S registradas en la base de datos SIH/SUS como Fracturas de Fémur, según la 10ª revisión de la Clasificación Internacional de Enfermedades (CIE-10) contenida en el capítulo XIX, Traumatismos, intoxicaciones y otras consecuencias de causas externas. En un análisis se incluyeron todos los códigos relacionados con las fracturas femorales (S72), sin especificar la localización anatómica. En el segundo análisis, solo se incluyeron códigos específicos $(720,721,722)$ referidos a fracturas de fémur proximal. La proporción de muertes hospitalarias entre ancianos se analizó a través de la relación entre el número de ancianos hospitalizados por fractura de fémur con el resultado de muerte por el número de ancianos hospitalizados por fractura de fémur. Los archivos fueron transferidos al programa de tabulación TAB para Windows (TabWin) para el análisis de datos. Se utilizó estadística descriptiva y se calcularon las frecuencias absoluta ( $\mathrm{n}$ ) y relativa (\%) del número de hospitalizaciones para cada variable.

Como los datos recopilados son de dominio público, están disponibles electrónicamente y sin la identificación de los participantes de la investigación, este estudio no requirió apreciación y aprobación por parte del Comité de Ética en Investigación. 


\section{RESULTADOS}

En Roraima, la proporción de ancianos hospitalizados entre 2015 y 2019 fue del 11,4\% (23.676). De este total, los ancianos hospitalizados por fractura femoral representaron el 2,4\% (578). El sexo femenino fue el más prevalente en las hospitalizaciones por fractura femoral (56,4\%). Estratificado por grupo de edad, 80 años y más representó el 38,7\% de las hospitalizaciones, siendo más prevalente en mujeres (69,6\%) (Tabla-1). Cuando se analizaron las hospitalizaciones, especificando la localización anatómica en fractura del fémur proximal, las mujeres representaron la mayoría (60,6\%). Estratificado por grupo de edad, 80 años y más, representó el 44,7\%, con la mayoría de las mujeres (70,3\%) (Tabla-2). La ocurrencia de hospitalizaciones por fractura femoral entre los ancianos con el resultado de muerte representó el 5,2\% (30), con la mayoría de las mujeres de 80 años o más $(66,7 \%)$

Tabla - 1 Hospitalizaciones por fractura femoral (sin diferenciar la localización anatómica) por grupo de edad y sexo. Roraima, Brasil, 2015 a 2019

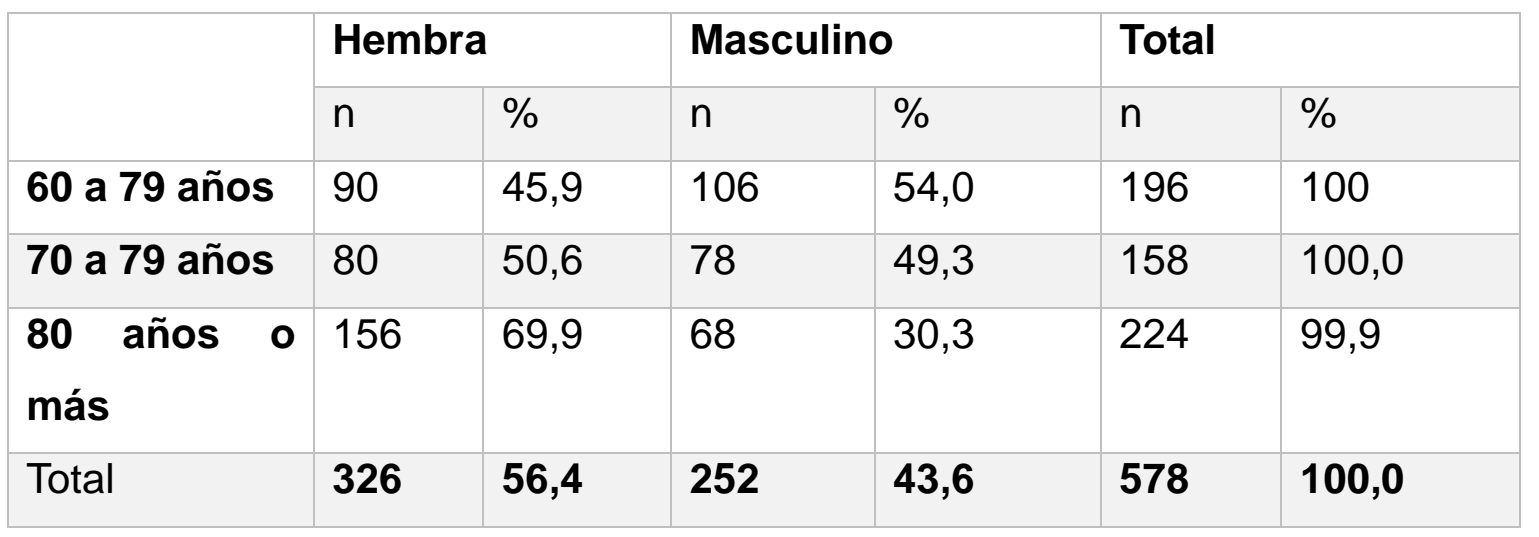

Fuente: SIH/SUS, DATASUS.

Tabla - 2 Hospitalizaciones por fractura femoral (localización anatómica del fémur proximal) por grupo de edad y sexo. Roraima, Brasil, 2015 a 2019.

\begin{tabular}{|l|l|l|l|l|l|}
\hline \multicolumn{2}{|l|}{ Hembra } & \multicolumn{2}{l|}{ Masculino } & \multicolumn{2}{l|}{ Total } \\
\hline $\mathrm{n}$ & $\%$ & $\mathrm{n}$ & $\%$ & $\mathrm{n}$ & $\%$ \\
\hline
\end{tabular}

RC: 105706

Disponible: https://www.nucleodoconhecimento.com.br/salud/fractura-femoral 


\begin{tabular}{|l|l|l|l|l|l|l|}
\hline $\mathbf{6 0}$ a 79 años & 33 & 54,1 & 28 & 45,9 & 61 & 100,0 \\
\hline $\begin{array}{l}\mathbf{7 0} \text { a 79 años } \\
\begin{array}{l}\mathbf{8 0} \text { años o } \\
\text { más }\end{array}\end{array}$ & $\mathbf{7 3}$ & 51,5 & 31 & 48,4 & 64 & 100,0 \\
\hline Total & $\mathbf{1 3 7}$ & $\mathbf{6 0 , 6}$ & $\mathbf{8 9}$ & $\mathbf{3 9 , 3}$ & $\mathbf{2 2 6}$ & $\mathbf{1 0 0 , 0}$ \\
\hline
\end{tabular}

Fuente: SIH/SUS, DATASUS.

\section{DISCUSIÓN}

Según los registros del SIH/SUS, de 2015 a 2019, el Capítulo XIX, Lesiones, envenenamiento y otras consecuencias de causas externas, ocupó la sexta posición entre las causas de hospitalizaciones entre los ancianos en Roraima, Brasil. A partir de este capítulo, la fractura femoral representó la principal causa de hospitalización.Este tipo de fractura es una de las lesiones traumáticas más comunes en los ancianos, y el avance de la edad y el género influyen en el aumento de la ocurrencia en esta población (WU et al., 2019; ARMOND et al., 2014; TSUDA, 2017).

Los resultados encontrados en este estudio están en línea con otros estudios que indican una mayor frecuencia de hospitalizaciones por fractura femoral entre las mujeres mayores (PORTO et al., 2019; OLIVEIRA e BORBA, 2017). Sobre las hospitalizaciones por fractura femoral con localización anatómica proximal, el perfil encontrado fue similar, corroborando otros estudios (NETO et al., 2011; PAULA et al., 2015). En su mayor parte, este tipo de fractura requiere hospitalización para tratamiento quirúrgico y presenta un mayor riesgo de morbilidad y mortalidad y una menor calidad de vida (PAULA et al., 2015; ALMEIDA et al., 2017).

Existe una relación entre el aumento de los casos de fracturas femorales y la edad avanzada, siendo el sexo femenino el más afectado (BORTOLON et al., 2011). Se sabe que debido a la deficiencia de estrógenos, las mujeres menopáusicas tienen una mayor incidencia de osteoporosis en comparación con los hombres. Por lo tanto, las fracturas de cadera, columna vertebral y muñeca son más susceptibles (CHEN et 
al., 2018). La disminución de la densidad ósea, la edad, los antecedentes de fractura, el tabaquismo, el consumo de alcohol y la artritis reumatoide son los principales factores de riesgo asociados con las fracturas en mujeres posmenopáusicas (WU et al., 2019; TSUDA, 2017). Los análisis mostraron que el nivel socioeconómico y el bajo nivel educativo también son factores para la aparición de la primera fractura de las estructuras de la cadera, como el fémur proximal (CHEN et al., 2018). Además, estos datos pueden retratar la feminización del fenómeno del envejecimiento de la población (ARMOND et al., 2014). Esto se debe principalmente a las diferencias encontradas en la esperanza de vida entre los géneros, y en el promedio general, las mujeres viven más tiempo que los hombres (TRAVASSO et al., 2020).

Las hospitalizaciones con el resultado de muerte por fractura femoral fueron mayores entre las mujeres mayores, según algunos estudios epidemiológicos (BORTOLON et al., 2011; PORTO et al., 2019). Se observó una asociación entre individuos mayores y un aumento en la tasa de mortalidad por fractura femoral. Sin embargo, algunos estudios muestran una prevalencia en la mortalidad entre los hombres mayores (PETERLE et al., 2019; OLIVEIRA y BORBA, 2017). La mayor proporción de mujeres hospitalizadas por fractura femoral podría justificar la prevalencia de muertes hospitalarias en este grupo. Sin embargo, un estudio realizado entre 2008 y 2018 sobre la mortalidad hospitalaria en las regiones de Brasil mostró una mayor mortalidad entre los hombres hospitalizados. También se observó que los costos resultantes de esta enfermedad son altos y presentan diferencias entre las regiones brasileñas (PETERLE et al., 2019). Los desacuerdos entre los hallazgos pueden estar relacionados con numerosas variables derivadas de la geografía y el clima, los hábitos alimenticios, las acciones de salud, los factores de riesgo, la constitución de la población y el subregistro de casos (SOARES et al., 2014). Otros factores de riesgo implicados pueden ser la presencia de más comorbilidades, alto riesgo quirúrgico en individuos de este grupo de edad, presencia de deterioro cognitivo y de sexo (EDELMUTH et al., 2018; NETO et al., 2011). 
El fenómeno del envejecimiento puede aumentar la incidencia de fracturas femorales si no se adoptan medidas preventivas (BEER y GILES, 2005). Además de un mayor riesgo de mortalidad, generalmente después de la aparición de la fractura, los ancianos tienen algún nivel de limitación física, deterioro de la salud mental y reducción de la esperanza de vida (EDELMUTH et al., 2018; OLIVEIRA y BORBA, 2017; ALEXIOU et al., 2018).

La distribución de la población anciana no es homogénea en Brasil, con diferencias regionales. La Región Norte tiene la menor concentración de ancianos, y el 53,2\% está representado por mujeres (TRAVASSO et al., 2020). A pesar de ello, el envejecimiento poblacional debe ser considerado como uno de los grandes retos para el sistema sanitario público, que no está preparado para atender esta nueva demanda. Se deben adoptar estrategias como la educación sanitaria y la prevención de fracturas para reducir los impactos negativos de esta enfermedad. Además, el uso de datos secundarios puede ayudar en el conocimiento de las necesidades del Estado y servir de apoyo en estrategias enfocadas en las demandas de salud.

Las principales limitaciones de este estudio están asociadas con el uso y la calidad de los datos secundarios disponibles en el SIH/SUS, como la subestimación de los casos y la imposibilidad de conocer las causas que llevaron a las hospitalizaciones por fracturas femorales. Además, no se utilizaron datos de población, lo que requiere estudios futuros más completos, principalmente debido a las características demográficas del Estado de Roraima.

\section{CONSIDERACIONES FINALES}

Según la edad y el sexo, se observó que las hospitalizaciones y muertes hospitalarias por fractura femoral fueron más prevalentes en mujeres octogenarias. Las estrategias de salud, como la prevención de caídas, deben dirigirse a la población de edad avanzada, especialmente a las mujeres, que son las más afectadas por esta enfermedad.Además, se deben adoptar acciones conjuntas dirigidas a mejorar el bienestar y la calidad de vida para un envejecimiento más activo y saludable, siempre teniendo en cuenta la realidad y las demandas locales. 


\section{REFERENCIAS}

ALEXIOU, K. I. et al. Quality of life and psychological consequences in elderly patients after a hip fracture: a review. Clin Interv Aging. v. 24, n. 13, p. 143-150, 2018. doi: 10.2147/CIA.S150067

ALMEIDA, D. O. et al. Internações por fraturas ósseas pelo SUS em idosos do município de Salvador (BA): um estudo descritivo do ano de 2015. Rev. Ciênc. Méd. Biol. v. 16, n. 3, p. 288-295, 2017. doi: 10.9771/cmbio. v16i3.24384

ARMOND, J. E. et al. Caracterização de idosos internados por queda acidental na cidade de São Paulo, 2013. Rev. Bras. Geriatr. Gerontol. v. 8, n. 4, p. 200-203, 2014.

BEER, C.; GILES, M. Hip fracture Challenges in prevention and management. Aus.Fam.Physician. v.4, n. 8, p. 673-676, 2005.

BORTOLON, P.C. et al. O perfil das internações do SUS para fratura osteoporótica de fêmur em idosos no Brasil: uma descrição do triênio 20062008. Cad. Saúde Pública. v. 27, n. 4, p. 733-742, 2011. doi: 10.1590/S0102311 X2011000400012

CARMO, W.M.; GOMES, L. S. M. Fraturas Trocantéricas. J Bone Joint Surg. v. 90, p. 700-7007, 2008.

CHEN, F. P. et al. Risk factors and quality of life for the occurrence of hip fracture in postmenopausal women. Biomed J. v. 41, n. 3, p. 202-208, 2018. doi: 10.1016/j.bj.2018.04.001

EDELMUTH, S. V. C. L. et al. Comorbidities, clinical intercurrences, and factors associated with mortality in elderly patients admitted for a hip fracture. Rev. bras. ortop. v. 53, n. 5, p. 543-551, 2018. doi10.1016/j.rboe.2018.07.014 
MACEDO, G. G. et al. Fraturas do fêmur em idosos: um problema de saúde pública no Brasil. REAC. v. 6, n. 1112, p. 01-07, 2019. doi: 10.25248/reac. e1112.2019

NASCIMENTO, M.M. Queda em adultos idosos: considerações sobre a regulação do equilíbrio, estratégias posturais e exercício físico. Geriatr. Gerontol. Aging. v. 13, n. 2, p. 103-110, 2019.

NETO, J. S. H. et al. Características epidemiológicas e causas da fratura do terço proximal do fêmur em idosos. Rev Bras Ortop. v. 46, n. 6, p. 660-667, 2011. doi: 10.1590/S0102-36162011000600007

OLIVEIRA, C. C.; BORBA, V.Z.C. Osteoporosis in brazilian patients awaiting knee arthroplasty. Acta Ortop Bras. v. 25, n. 4, p. 155-158, 2017. doi: 10.1590/1413-785220172504168827

PAULA, F. L. et al. Readmissão e óbito de idosos com alta após internação por fratura proximal de fêmur, ocorrida nos hospitais do Sistema Único de Saúde entre os anos de 2008 e 2010. Ver Bras Epidemiol. v. 18, n. 2, p. 439-453, 2015. doi: 10.1590/1980-5497201500020012

PETERLE, V.C. U. et al. Indicators of morbidity and mortality by femur fractures in older people: a decade-long study in brazilian hospitals. Acta Ortop Bras. v. 28, n. 3, p. 142-148, 2020. doi:10.1590/1413-7852202028032283

PORTO, A. O. et al. Características sociodemográficas e custo de hospitalizações por fratura de fêmur em idosos na Bahia. J Health NPEPS. v. 4, n. 2, p. 297-309, 2019. doi: 10.30681/2526101038233823

SANTOS, R. B. et al. Older adults' views on barriers and facilitators to participate in a multifactorial falls prevention program: Results from Prevquedas Brasil. Archives of Gerontology and Geriatrics. v. 92, p. 01-09, 2021. doi:10.1016/j.archger.2020.104287 
SOARES, D. S. et al. Fraturas de fêmur em idosos no Brasil: análise espaçotemporal de 2008 a 2012. Cad. Saúde Pública. v. 30, n. 12, p. 2669-2678, 2014. doi: 10.1590/0102-311X00218113

TRAVASSO, G.F. et al. The elderly in Brazil: demographic transition, profile, and socioeconomic condition. Rev. bras. estud. popul. v.7, p. 01-27, 2020. doi: 10.20947/S0102-3098a0129

TSUDA, T. Epidemiology of fragility fractures and fall prevention in the elderly: a systematic review of the literature. Curr Orthop Pract. v. 28, n. 6, p. 580-585, 2017. doi: $10.1097 / B C O .0000000000000563$

WU, S.C. et al. The influence of ageing on the incidence and site of trauma femoral fractures: across-sectional analysis. BMC Musculoskelet Disord. v. 20, p. 413-419, 2019. Doi: 10.1186/s12891-019-2803-x

\section{APÉNDICE - NOTA AL PIE}

[2] Sistema de Informações Hospitalares (SIH).

[3] Sistema Único de Saúde (SUS).

[4] Autorizações de Internações Hospitalares (AlH'S).

Enviado: Agosto de 2021.

Aprobado: Enero de 2022. 